\title{
Semantic Modeling of Internal Audit Field
}

\author{
Stamatios A. Theocharis and George A. Tsihrintzis
}

\begin{abstract}
The constantly changing economic environment, in which businesses and public sector organizations in recent years grow and operate, holds risks associated with competition, economic uncertainty and other inherent business risks. Therefore we speak of the need of identifying and managing those risks. Important role in this field is played by the internal audit and internal control systems. This field, running through all the activities of public and private sector bodies while distinguished by the highly complicated methods of operation and organization. To assist the administrations in the management of the audit mechanisms modern and appropriate information systems are required. It is appropriate in this work, as a key step in the field of study to list the relevant concepts and to model the entire system in the form of an OWL ontology. Also present examples of query ontology using Semantic Web technologies and the conclusions reached.
\end{abstract}

Index Terms-Audit ontology, internal audit, internal control.

\section{INTRODUCTION MOTIVATION}

Businesses and public sector organizations face in recent years a very complex and competitive environment which hinders their operation and further development. This environment is characterized by volatile financial figures and the existence of specific risks arising either by increasing global competition or other economic or inherent operational factors. There are, however, risks associated with errors or non-normal situations related to the business or the organization itself. In the area of dealing with these risks, we consider that the development of the auditing science and in particular the internal audit is of great importance. The control does no longer concerns only the financial figures of a company or organization but also the compliance with the policies espoused, the legality of the acts of employees and finally the prevention of corruption phenomena.

The concept of internal audit concerns the monitoring and evaluation of the overall operation of enterprises and organizations "from the inside" and is an essential tool of the authorities in their attempt to shield their services from voluntary or involuntary acts detrimental acts, and behaviors which have a negative impact on the organization's interests. For this purpose the administrations of organizations usually adopt an Internal Control System as a set of operational actions for the purpose of their effective, efficient, and safe operation. By the Internal Control System specific procedures and methods of operation or production of goods and services are set out and specific safeguards for their compliance are provided. In the public sector this is already being addressed systematically through the institutional framework - legislation, circulars, and directives - defining the function of public sector entities. In the private sector this is the responsibility of each administration and varies according to the size and scope of business.

The compliance with the rules set by the Internal Control System, and any inconsistencies or failures are subject to relevant controls of internal audit units. These services are typically staffed and operate within the organization itself and aim to assure the administration about the adequacy and consistency of the Internal Control Systems that have been adopted. Their operation concerns all the entity's functions, and covers both administrative matters as well as productive and financial matters. In recent years, the ubiquitous use of information systems and the Internet, has led the Internal Audit to extend to the field of Information Technology Governance, which is of particular interest.

Motivation for this work has been the activation of Internal Audit Units in the public sector entities in Greece, about three years ago although Greek government had institutionally planned to operate them in the public sector since 2006 [1]. Related reports regarding the listed companies exist in Greece since the beginning of 2002 [2]. Internal audit is for the Greek Public Administration a new operating area in which special attention should be paid to the administrative and functional integration and the further consolidation and development. Especially for the authors of this paper, this is an area of concern as the internal control is associated with the processes and the information systems concerning the application of e-government.

In this paper an attempt is made to map the environment of internal audit in terms of semantic webby using relevant tools, like Protégé. This was considered appropriate since the audit environment and the procedures followed in the internal audit units are particularly complicated and combine various cognitive objects. The study of internal audit as an object is summarized in the second part, as an essential structural part of this work. Then, by using a special tool we implemented specific ontology in order to record the terminology and the corresponding concepts and entities that characterize the field of internal audit. The reasoning and the result of this work, is presented in the third part. In the fourth part we present the results of semantic search with the help of relevant tools and in the fifth part we evaluate the ontology developed by using relevant tools and in the sixth part we give the overall conclusions of our work.

\section{PResentation Of The Audit Field}

Manuscript received January 25, 2017; revised March 30, 2017.

Stamatios Theocharis and George Tsihrintzis are with the Dept. of Informatics, University of Piraeus, Greece (e-mail: stheohar@unipi.gr, geoatsi@unipi.gr).

\section{A. Generally about Audit}

Auditing as a branch of economics and management is both science and technique. It is characterized as science 
because through the investigation, it constantly generates new knowledge and as technique because along with the scientific knowledge generated, it meets the needs of audit thus achieving its mission.

More specifically by the term auditing we mean the systematic process of gathering and evaluating audit evidence from an independent, capable, and appropriate person. In greater detail, the content of the auditing may be categorized into the following three themes:

- The audit object, ie what is audited, why it is necessary to carry out an audit and what objectives are pursued and achieved through audit.

- The subject of the audit (auditors) ie which persons perform auditing and what should be their qualifications, their skills and the necessary experience.

- The auditing procedures (methodology and technique of audit), ie how the audit is carried out.

\section{B. Audit Categories}

The controls are divided, according to various criteria, in the following main categories [3].

- Internal audits are the audits organized by the body itself and carried out by qualified and properly trained executives, internal auditors, who are employees of the organization.

- External audits, are the audits performed by external auditors who have no employment relationship or employee status with the body that they are invited to audit.

- Mixed audits are organized and coordinated by the Internal Audit Units and carried out in collaboration with external auditors.

- Special audits are those who are exploring a specific field or object, such as procurement, revenues, payments, inventory management, quality assurance procedures of all the Agency's activities etc.

- General audits are those extending across the management for a specified period.

- Preventive audits are those which aim to prevent the risk and are carried out by the same organizational units involved every time.

- Repressive audits are those carried out subsequently. They aim at testing the implementation of procedures and the confirmation that the objectives were achieved in full in an effective and efficient manner. They also aim at revealing and suppressing errors and omissions, irregularities, theft and mismanagement of funds and resources.

- Permanent or ongoing audits are those conducted on an ongoing basis and take place during the execution of the financial act, transaction or productive operation.

- Regular or periodic audits are those carried out at regular periods.

- Temporary or occasional audits are those carried out in exceptional cases either by the internal audit service initiative or by order of the Administration or following specific complaints. These are audits carried out after assessing that there has been suspected waste, abuse, theft or corruption and generally wherever failures identified in the functions and immediate determination of their size is required in order to take administrative measures.

\section{Concept and Internal Audit Content in Public Administration}

Internal audit should not be confused with the Internal Control System. Usually, an internal control system adopted by the management body in order a) to protect the company's assets b) to secure and check the accuracy and reliability of its accounting data c) to improve the effectiveness and efficiency of its operations and d) the compliance and implementation of its operational policies. According to [4], an internal control system has specific elements components that make it strong [5]. a) Audit environment as the foundation for all other components, setting the stigma and the general spirit of the enterprise. The factors that affect it are the integrity, the moral values, the abilities and skills of the organization's people, etc. b) Risk assessment. A prerequisite for risk assessment is establishing objectives at different levels of the organization that are linked to each other and are internally coherent. The risk assessment lies in identification and risk analysis regarding the achievement of goals and objectives of the organization and determining how they should be managed. c) Auditing activities are all the policies and procedures that ensure the compliance with the instructions of the organization's administration, d) Information and communication. That is, all the tools to manage and disseminate information which assist in making business decisions, e) Monitoring. That is, the quality assessment procedures of system performance.

On the other hand, the internal audit is an independent, objective, guaranteeing and consulting activity designed to add value to the organization and improve its functions [6]. It also contributes to achieving the organization's objectives through a systematic method of valuation and improving the efficiency of processes, risk management, internal control and corporate governance.

The internal control can and should control all Directorates, Departments, services, functions, activities as well as policies, procedures, regulations, and applied practices that constitute the internal control system of the institution. It is a tool of the Administration, which enlightens, advises and guides aimed at overall improvement. It intervenes to examine a process or an activity of the organization and deposits a) a diagnosis confirming more or less the good operation, b) a prognosis to alert those responsible for the central administration and c) a treatment that aims to ensuring the organization's interests, the reliability of information, the effectiveness of operations and the competitiveness.

The primary purpose of internal audit is to assist all executives of the organization (at all levels of administration) to effectively perform their duties and in this way to provide estimates, recommendations, opinions and information relating to the activities in question. Its object is to examine and evaluate the adequacy and implementation of various functional systems an organization and their control systems, as well as the examination of the quality of the organization's actions within the framework of undertaken responsibilities. In addition, auditors should protect the essential values of public administration as they serve all citizens. The auditing procedures as formulated by the Institute of Internal Auditors [6], adapted to the nature and the public sector scope are:

- Oversight. The auditors help the directors to supervise whether the public sector services do what they ought to 
do, if they use the available funds for the intended purposes and if they comply with the laws and regulations.

- Detect. The detect aims to identify improper, inadequate, illegal, fraudulent or abusive acts already done and to obtain the information that will lead to decisions related to criminal prosecutions, disciplinary actions, or other corrective actions.

- Deterrence. Deterrence is designed to identify and limit the circumstances that allow corruption.

- Insight. The controllers provide insight to help managers to determine which programs and policies work and which do not.

- Foresight. The auditors help organizations have foresight identifying future trends and focusing their attention on the potential challenges before they grow into crises.

\section{Audit Types}

The types of Internal Audit are:

- Production Audits

- Financial Audits, which comprise the auditing procedures relating to the security of the business's wealth and assets and aim to verify the accuracy, honesty and reliability of financial statements and data and identify its legality.

- Operational audits, which include the framework and the assessment procedures on the compliance with the policies and the company's operating procedures. The objective of operational audits is the evaluation and assessment of the existing system structure and the proposal to develop new systems and improve existing ones in areas that have been discredited.

- Management audits, which include the organizational framework and the procedures related to decision making, compliance with them and their evaluation. In contrast to the financial and operational audits, they have broader objectives which consist of examining and evaluating, based on objective and scientific methods, the overall administrative efficiency of the business/body.

- Information technology and information systems audits. They aim to establish the extent to which reliability, confidentiality and integrity of information available is ensured. These lie in the effectiveness of information systems, the proper use of resources, the strengthening of infrastructure and information security, the correctness, completeness and accuracy of transactions and the updating of information files.

\section{Modeling of KNOWLEdge Within the Auditing SECTOR}

The knowledge of the organization's structure and the risks of which it is threatened, the internal controls designation and proper functioning, the auditing procedure, reports issuing to the management and continuous monitoring of the organization's work. All the above are the main work areas of an Internal Audit Unit. This consideration, however, must be continuous, since thing often change, especially safeguards, the person authorized to apply them, the organization risks and the organization's objectives. A prerequisite for achieving the above is the retrieval of information in innovative ways and then the effective management of knowledge generated by them. It is therefore an important factor of success for the internal audit unit, the modeling of the overall environment in a semantic approach. As a primary step in the study of audits' environment and in order to create a knowledge base, we considered appropriate, to present in this work, the recording of key concepts and entities of the audit as well as the properties and the relationships that characterize them. The properties and relationships between concepts and entities are essential for the performance of semantics in concepts and individuals in this database and constitute the key difference from a relational database. This database becomes useful in many ways, including a) the ability to directly semantic search for information based on the relationships between classes and between individuals, b) as an educational material for those involved with the audits, c) visualization of a complex system of concepts, entities and associations, d) future use of the base as an input file to generate associated data. This was made possible by the modeling of the relevant knowledge of the auditing field in the form of an OWL ontology. For this purpose, we used the application Protégé 5.1.0 [7]. The ontology we present is equipped with all the basic concepts, with some corresponding virtual individuals and the properties that interconnect (Fig. 1). Also we defined, where necessary, restrictions on the domain and range of classes and specific inference rules in SWRT tool of Protégé.

In particular, we present the most essential classes we defined and their respective subclasses in correspondence with the actual concepts and their relationships, as discussed above.

\section{A. Definition of Classes}

- Classes "Audit" and "Auditors" resulting from the basic entities of the field of interest: audits and auditors respectively while the physical entities constitute the corresponding individuals of the related classes.

- The class "AuditDescriptor" was set to correspond to the characteristics that describe the audits. Here we have defined as subclasses of "AuditCategory" and "AudiType" to classify the corresponding instances of audits in the appropriate categories and genres. Also we have set the remaining subclasses shown in Fig. 2 by formatting the assumption that "any audit - as an individual - belongs to at least one category ("AuditCategory"-class) and type ("AuditType"- class), has a specific object ("AuditObject"-class), while based on a specific plan ("AuditPlan"-class) and function ("AuditPerformance"class) it produces effects ("AuditOutput"-class), fulfilling the purpose ("AuditPurpose"-class) specified ("has Assignor"- object Property) by the assignor ("Assignor"-class)".

- Similarly we have set the class "AuditorDescriptor" in order to format the characteristics of each auditor (Fig. 3) : "each auditor ("Auditor"-class) is classified into a specific category ("AuditorCategoryByObject”- class) related to his object, has specific educational characteristics ("AuditorEducation"-class), special training ("AuditorTraining"-class), experience (“AuditorExperience"-class) and skills ("AuditorSkills"class)"

- An audit is an individual of the Audit class and 
simultaneously one of the classes that describe audit. For this reason the "Audit" and "AuditDescriptor" classes are not disjoined to each other. For example, the control of the procedure for obtaining a citizenship decision

- "AuditRelatedToCitizenship" is individual of "Audit" class, "Regular" class and "InternalAudit" class. Similarly, each auditor (physical entity), is an individual of the class Auditor and simultaneously the "AuditorCategoryByObject" class as a subclass of "AuditorDescriptor"

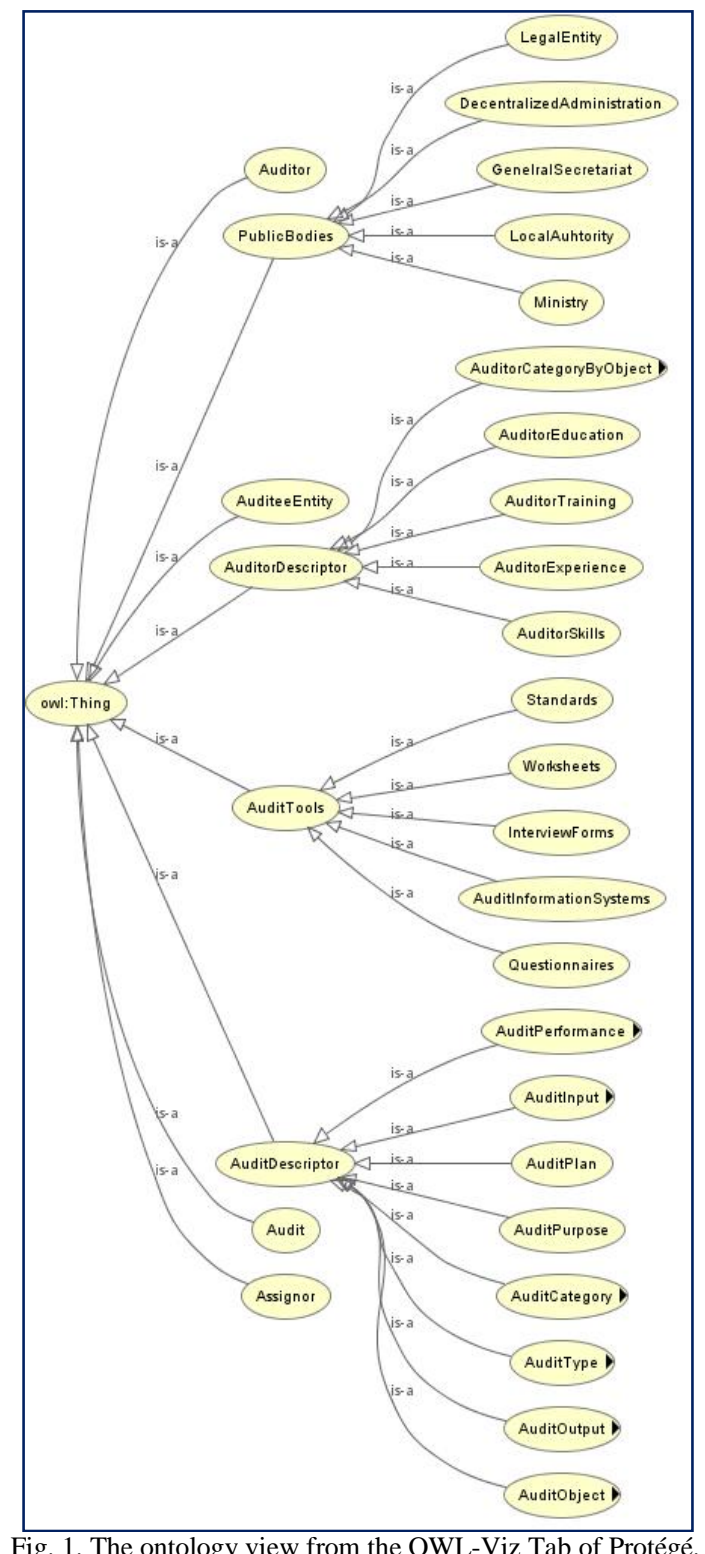

- As each audit is mandated by the responsible body, we defined as base the "Assignor" class which has as individuals all the assignors who commands the carrying out of the audits. Also as in each audit specific tools are used, such as Information Systems, Interview Forms, Questionnaires and Worksheets, we defined the corresponding classes with individuals the instances of each case.

- As each audit refers to one auditee entity and that entity belongs in the public body we respectively defined the classes "AuditeeEntity" and "PublicBodies". Each auditee entity constitutes an individual of the
"AuditeeEntitee" class while the particular public sector organizations are represented as subclasses of the "PublicBodies" class.

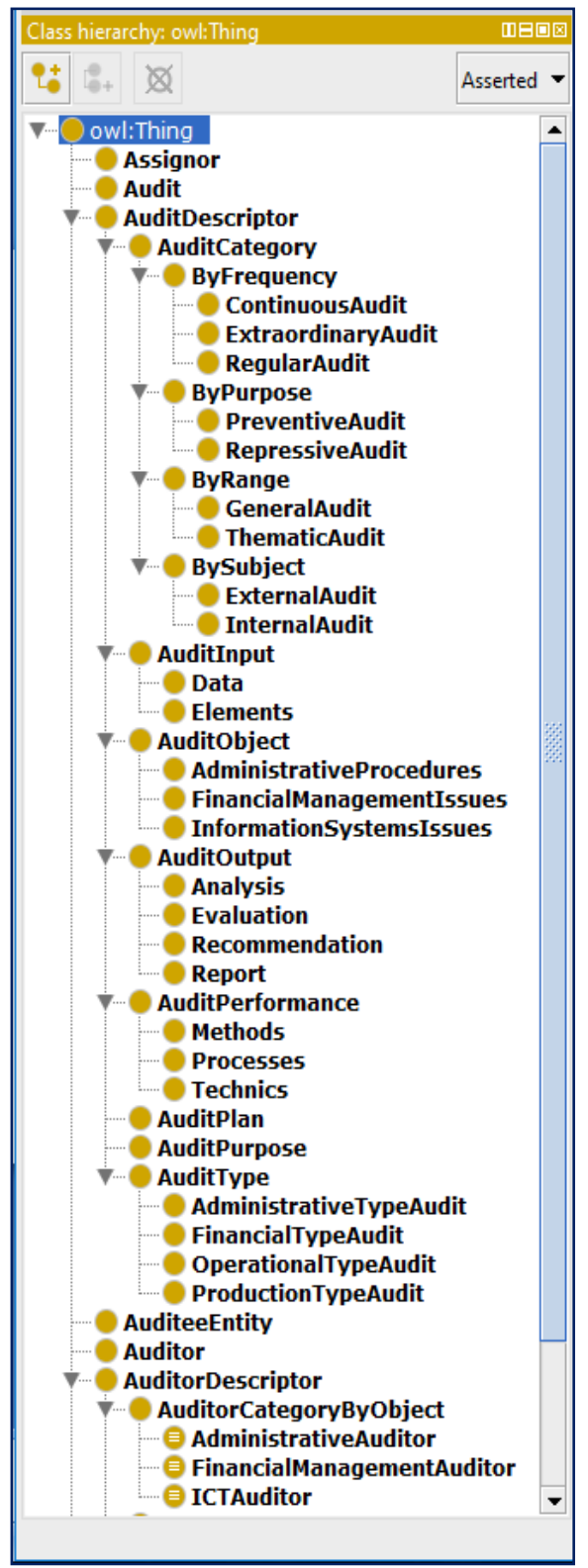

Fig. 2. The ontology class hierarchy in Protégé.

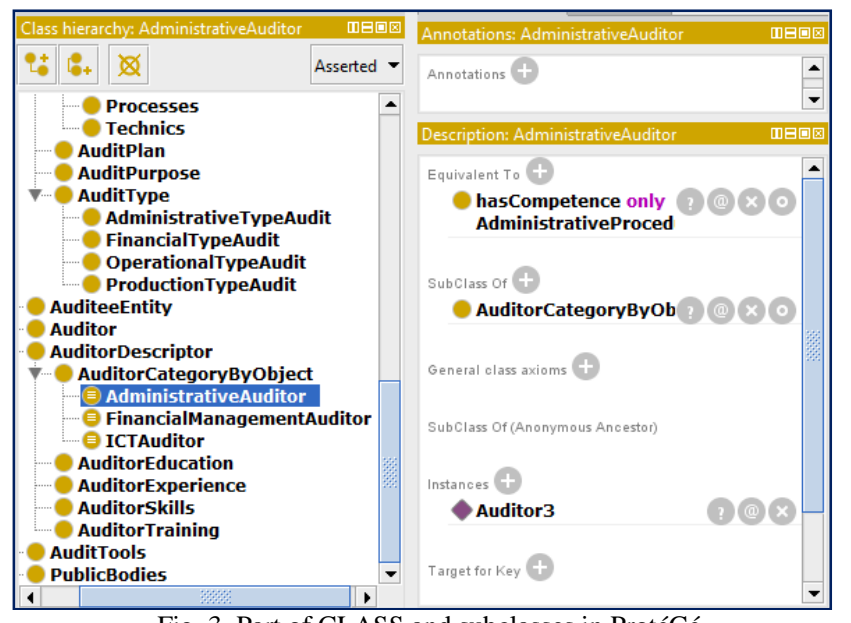

Fig. 3. Part of CLASS and subclasses in ProtéGé.

B. Definition of Properties 
The properties defined by the user are of particular interest as they appear in the corresponding tab (Fig. 4).

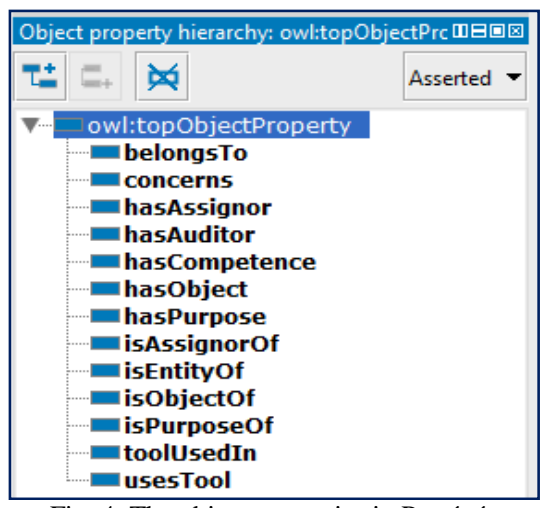

Fig. 4. The object properties in Protégé.

- In particular, we mention the properties "has Auditor" and "hasObject" that connect the audits with the auditors and their objects. These properties combined with the respective classes, perform semantically the knowledge that "each audit ("Audit"-class) carried ("hasAuditor"-object property) by an auditor ("Auditor"-class) and has a specific object ("hasObject"-object property)". Their use appears in the corresponding tab of Protégé (Fig. 5). Relative is also the property has Assignor, connecting the audits with their assignors, in order to perform the knowledge that "each audit ("Audit”-class) has ("has Assignor"-Object Property) an assignor ("Assignor"-class).

- Also interesting is the property "hasCompetence" which connects the "Auditor" class to the object controlled. Its use is shown in the corresponding tab of Protégé. It is used as an example to identify as "ICTAuditor" class any individual whose duties include matters relating to information systems (Fig. 6).

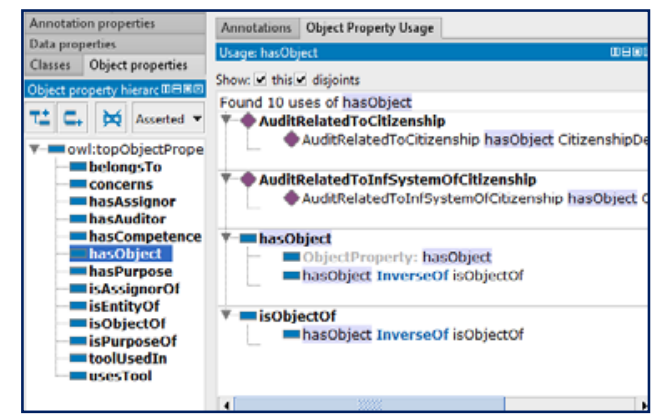

Fig. 5. The has object property and its use.

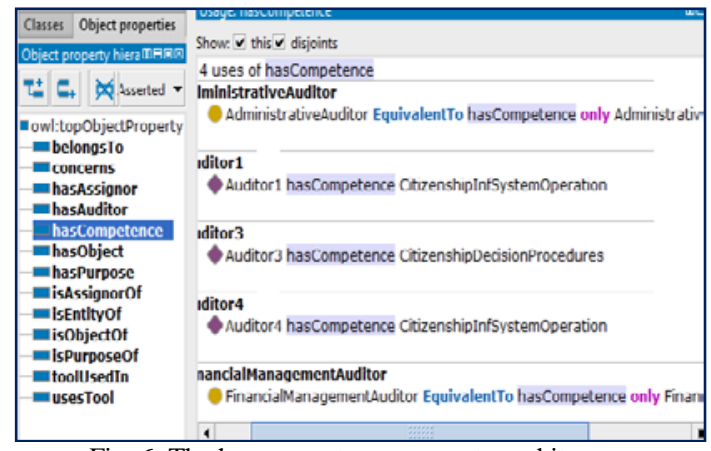

Fig. 6. The has competence property and its use.

- Useful property when searching for information in the field of audits is "usesTool", linking the audits and tools used to them. An example of the use of this property in the ontology ISO_27007 [11] particular tool is shown in (Fig. 7).

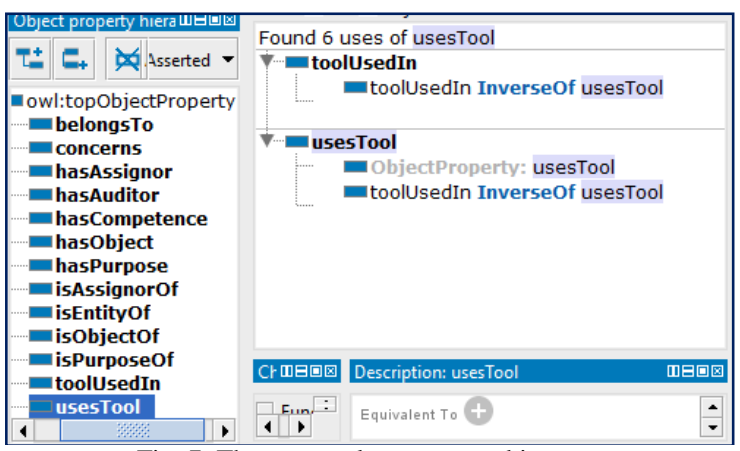

Fig. 7. The uses tool property and its use.

\section{EXAMPLES OF THE APPLICATION OF RESTRICTIONS, RULES AND QUERIES IN ONTOLOGY}

With our OWL ontologies we are given the opportunity to state the relationship between classes, with the definition of suitable properties within the logic of the data representation in RDF. For example we declared the property "hasObject" and we set up as a domain class "Audit" and range class "AuditObject". This way we declare the the reasoning that if the individual "AuditRelatedToCitizenship" is associated with the individual "CitizenshipDecisionProcedures" then these belong to Classes "Audit" and "AuditObject" respectively. In this way and by means of the reasoners provided by the application specific conclusions are produced as appropriate (Fig. 8).

In order to state specific restrictions on the members of classes of the ontology, we used the concept of equivalent class. This was achieved with the statement of logical expressions in OWL, which use properties and classes appointed by the user. For example in "ICTAuditor" class we stated as equivalent class the one derived by _ the logical expression "hasCompetence only InformationSystemsIssues" (Fig. 9). This declaration serves the need to reduce the members of the "ICTAuditor" class only to the members that satisfy the condition that "the information systems auditors have responsibilities only to information systems issues". If no such restriction is satisfied the systems of the reasoners of the application display a message for data inconsistency.

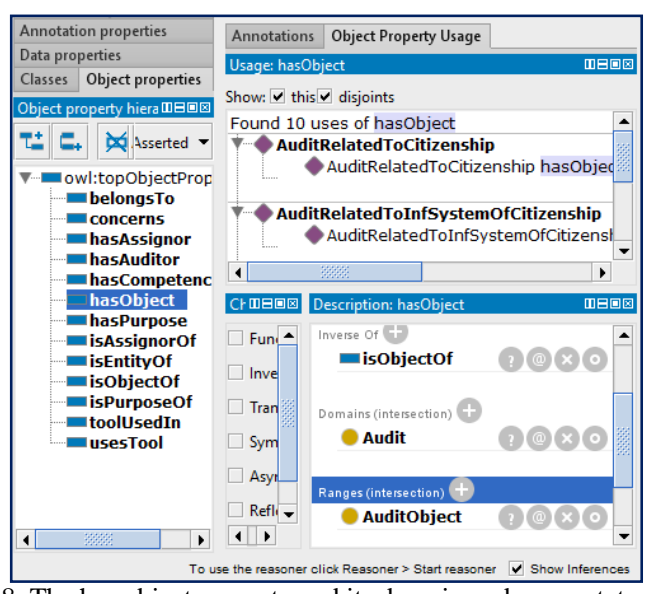

Fig. 8. The has object property and its domain and range statement. 


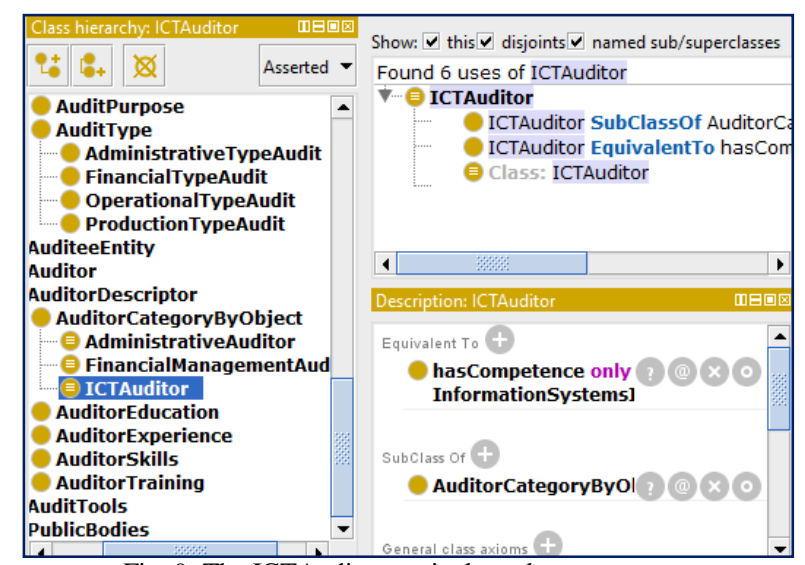

Fig. 9. The ICTAuditor equivalent class statement.

Another way to impose certain restrictions or to state facts which lead to the conclusions on the initial data is the SWRL tool offered by the Protégé. This tool uses the same language to designate the desired events which will form the basis of the inference in DL Query tool Protégé. For example in Fig. 10 , we have stated that "whoever is auditor of information systems, then belongs to the class of auditors"

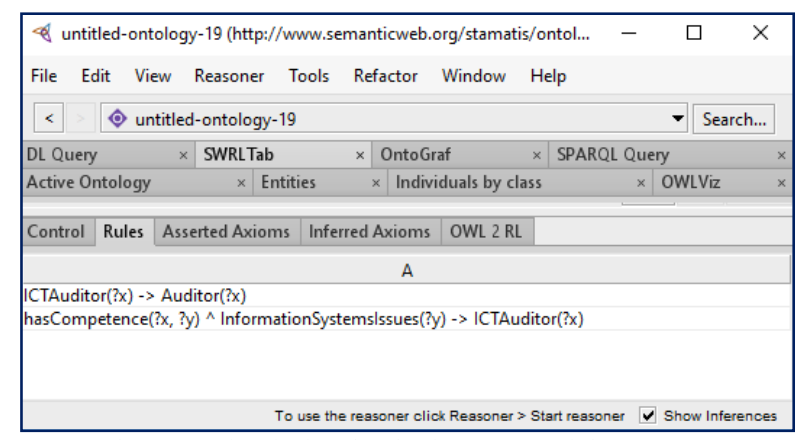

Fig. 10. Rules declaration in the SWRL tab in Protégé.

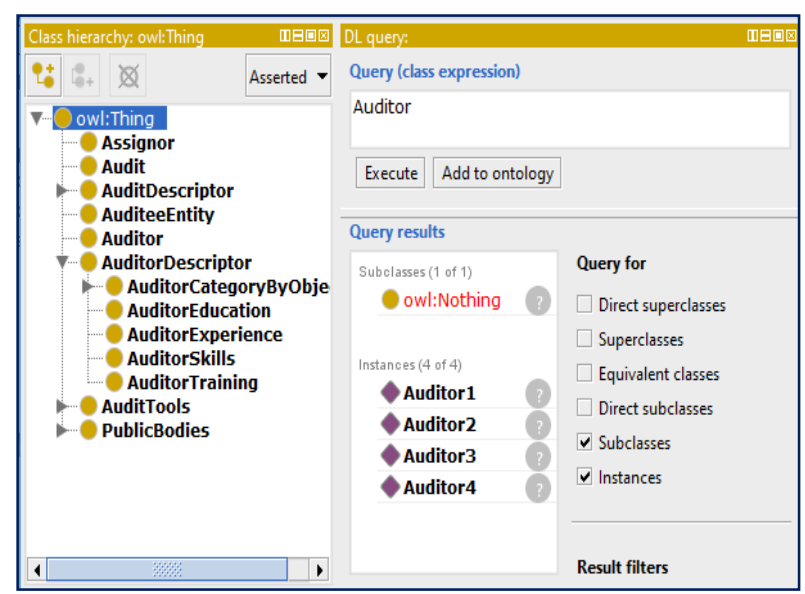

Fig. 11. The effect by the relevant query about the auditor class.

The Protégé provides two basic query tools for the ontology: the DL Query and the SPARQL environment. The first tool is based on the function of the reasoners also provided as plung-ins of the Protégé and uses inference supported by OWL on the stated properties. By using this tool information is produced that constitutes inference product, without directly resulting by the statement of classes, members and their properties. For example Fig. 11 shows the effect by the relevant question we posed to the ontology about the members of the "Auditor" class. Initially member “Auditor1", was not declared as a member of a class. By applying the inference of the reasoner and DL Query we see that the member "Auditor 1 " is conclusively member of the class "Auditor". In contrast, the SPARQL environment operates on the logic of the RDF data, and runs all the stated RDF triples without applying any other kind of inference. A typical result is shown in Fig. 12, where the "Auditor1" is not forming part of the results of the relevant search.

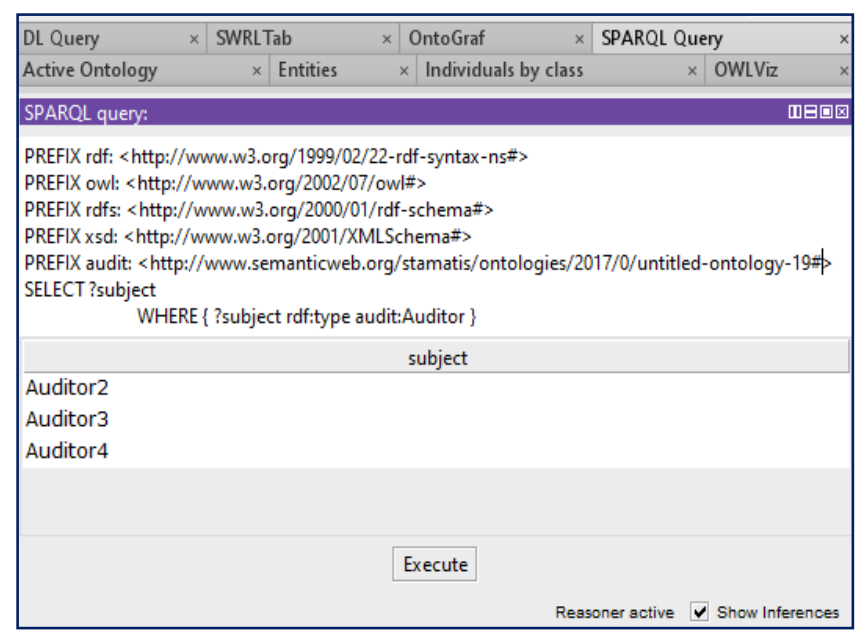

Fig. 12. The response of SPARQL query in Protégé.

\section{EVAluAtion Assessment OF ONTOLOGY}

In order to examine the level of consistency of the ontology under the actual circumstances of the audit, we recovered the official text of the standard ISO / IEC 27007: 2011 [11]. By this standard, we cover issues related to the planning of audits, the object, the frequency of audits the procedures to be followed and the qualifications of suitable auditors in the field of IT security. We also cover design and auditing content issues, auditing activities and compliance issues. All model's elements listed, are already included in the ontology developed as basic classes. Note that if judged necessary to add new concepts or properties, this does not affect the operation of the original version. We conclude that the ontology could be integrated into productive operation to be fed with real data.

Moreover, the Protégé offers through reasoners control mechanisms for the consistency and precision of ontology. Any problems are encountered during the running of the DL Query independently or by calling the specific reasoners. As shown above when running relevant DL questions, there were no inconsistencies in the ontology.

Regarding the valuation of the ontology we can mention the following advantages of the proposed modeling.

- Simplicity of stating classes, instances and relations between them

- Possibility of direct enrichment of the base without the requirement to change the existing structure from the outset.

- Consistency with truly international standards applying on audits

- Ability to query the basis of logical inferences

On the downsides we note the manual query process. However this issue can be addressed by appropriate programming techniques applied on the data that can be extracted from the ontology, which is not the subject of this paper. 


\section{CONCLUSIONS FUTURE WORK}

Due to constant changes in economic, entrepreneurial, or regulatory conditions, it has become urgent for the auditing bodies to adopt and use appropriate mechanisms and tools to identify and counter the risks associated with the expected changes. At the same time there is an enlargement of the audit environment, with the addition of an additional audit field, that which is related to the governance of information systems and electronic communications. As a consequence, we have a multiplication of the operational activities of audit services - internal or external on one hand and the need to modernize and / or full review information systems to support their work on the other. At the same time, the work of dissemination of information related to audits, in the higher hierarchical levels of organizations, where the decisions are made, must be supported.

Existing information systems used by the auditing bodies, are now facing their needs as appropriate and are based on traditional technologies of relational databases and decision support systems. The field described belongs to those cases that can be supported more successfully than the technologies of the Semantic Web, as it s characterized by the large volume of data which is still increasing, the various categories and types of information that change rapidly and must be shared with different rights to users. These features cannot be addressed in terms of resource economy with traditional programming techniques and tools. Moreover, this area is not characterized by strong commercial demand leading to a reduced supply of software applications. This was one of the motivations to investigate the possibility of software development based on semantic web technologies and associated data.

In order to meet the operational needs of the audit mechanisms of the public sector, the systematic study of the audit area is essential to develop a "smart" integrated information system that meets the real needs of the auditors. The issues that emerge in this study are the following.

- Diagnosis and identification of operational needs. To serve the work of audit mechanisms, we must take into account the differences of the audit environment between the various organizations, but also the similarities of the audit procedures followed by all internal audit units or other control mechanisms. In any case it is necessary to have common information search and management tools and resources, automated planning and control, measurement tools and risk assessment, task management tools, etc.

- Determination of the information system purpose. A common requirement of all control mechanisms is mainly to provide information. Specifically: a) the production, management and intelligent search of information materials relative to audits, such as operating manuals and legislation, b) auditing equipment like questionnaire forms, audit reports, check-lists c) analyzes of issues, findings and points of attention per audit category, as resulting from the corresponding reports.

- Access to the information system data. Key features are making the large volume of data and information that can constitute sources and/or audit result and the fact that some information may be public and others of special classification. For this reason it is necessary to use "smart" technical data and search interface. Such techniques are offered through the technologies of semantic web and linked data.

- Maintenance - management. The maintenance and management of a centralized system must be the subject of a central public service that will ensure the stability, confidentiality and integrity of information and to ensure proper operation.

\section{ACKNOWLEDGMENT}

This work has been partly supported by the University of Piraeus Research Center.

\section{REFERENCES}

[1] (2006). Law 3492. Greek Parliament. [Online]. Available: http: www.et.gr

[2] (2002). Law 3016. Greek Parliament. [Online]. Available: http: www.et.gr

[3] Conducting manual of internal audit, ministry of finance, Greece. [Online]. Available: http://www.publicrevenue.gr/kpi/

[4] (2014). Improving organizational performance and governance: How the COSO frameworks can help. [Online]. Available: https://www.coso.org/Documents/2014-2-10-COSO-Thought-Paper.p df]

[5] COSO. [Online]. Available: https://www.coso.org

[6] Theiia. [Online]. Available: www.theiia.org

[7] Protégé Project. Stanford University. [Online]. Available: http://protege.stanford.edu/

[8] Official site of American Institute of Certified Public Accountants-AICPA. [Online]. Available: http://www.aicpa.org/

[9] Modernizing Internal Audit, "Greece-OECD project on technical support on anti-corruption,” Report on the learning and consultation workshop, 2016, Athens, Greece.

[10] ISO Store.

[Online].

Available: http://www.iso.org/iso/home/standards/management-standards/iso270 01.htm

[11] (2011). ISO/IEC 27007: Information technology-security techniques-guidelines for information security management systems auditing. [Online]. Available: http://www.iso27001security.com/html/27007.html

[12] Standards-guidance. [Online]. Available: https://na.theiia.org/standards-guidance/mandatory-guidance/Pages/St andards.aspx

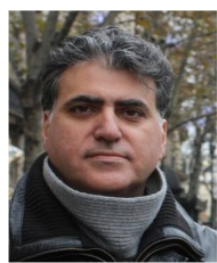

Stamatios A. Theocharis was born in Athens. He is a graduate of the Department of Mathematics, University of Ioannina-Greece and a post-graduate of the Department of Informatics, University of Piraeus-Greece. He is currently a Ph.D candidate in the domain of e-Government and open data analysis with his supervisor professor George Tsihrintzis. His current professional occupation concerns the object of the ministry of interior of Greece and particularly is the head of internal audit unit. open data, semantic web and the technologies that support it and the e-Government technologies are included in his research interests.

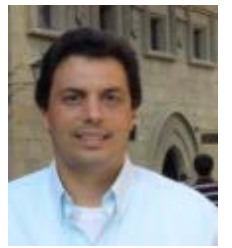

George A. Tsihrintzis is the president of the Department of Informatics in the University of Piraeus, member of the council, the director of graduate studies in 'advanced computing and informatics systems' and full professor in the University of Piraeus, Greece. He received the diploma of electrical engineer from the National Technical University of Athens, Greece (with honors) and the M.Sc. and Ph.D. degrees in electrical engineering from Northeastern University, Boston, Massachusetts, USA.

His current research interests include pattern recognition and machine learning and their applications in multimedia interactive services, user modeling, knowledge-based software systems, human-computer interaction and information retrieval. He has authored or co-authored over 300 research publications in these areas, which include 5 monographs and 13 edited volumes. 